\title{
MicroRNAs: Biomarkers or Therapeutic Targets?
}

ISSN: 2576-8816

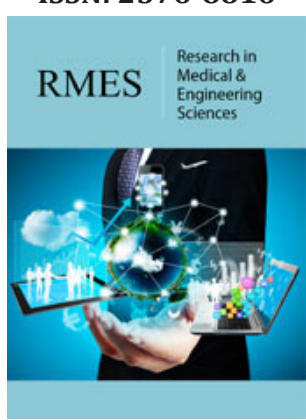

*Corresponding author: Areti Moushi, Cyprus Institute of Neurology and Genetics, Cyprus

\section{Submission: 侮 July 25, 2019}

Published: 侮 August 27, 2019

Volume 8 - Issue 2

How to cite this article: Mehmet A 0 . Mechanisms that Trigger Persister Resuscitation. Res Med Eng Sci. 8(2). RMES.000681.2019.

DOI: 10.31031/RMES.2019.08.000681

Copyright@ : Areti Moushi, This article is distributed under the terms of the Creative Commons Attribution 4.0 International License, which permits unrestricted use and redistribution provided that the original author and source are credited.

\section{Areti Moushi*}

Cyprus Institute of Neurology and Genetics, Cyprus

\section{Opinion}

MicroRNAs are small non-coding RNAs of approximately 22 nucleotides, that control gene expression. They pair with s specific part on the untranslated region (UTR) of their target mRNAs and silence their expression, either by inhibiting their translation or by degrading the sequence. Therefore, a dysregulation in the expression of miRNAs results to the dysregulation of their target genes which in several cases they may play in different diseases. In the past decades the investigation of these non-coding RNAs has been comprehensive and thorough. MiRNAs have been investigated for their possible role as biomarkers as well as therapeutic targets. The expression of a biomarker should be different between patients and individuals without the specific disease and ideally, they should be specific, sensitive and be collected via non-invasive techniques. MiRNAs seem to have these characteristics, since they are differentially expressed in different tissues and they can be collected through body fluids, such as blood, urine or saliva samples thus making then ideal as biomarkers. In addition, they can withstand extreme condition and be stable for long periods of times thus making them ideal as biomarkers. Cardiovascular diseases were investigated in depth, identifying miRNAs which are differentially expressed in patients compared with individuals without the disease which could potentially be promising biomarkers or prognostic factors. According to Zhou and colleagues (2018), approximately 30 miRNAs have been identified to be associated with heart failure, a condition that damages the heart muscles, which is one of the major causes of death in the United States. Additionally, in Acute Myocardial Infraction and Arrhythmia the expression levels of one and four miRNAs respectively were identified to be significantly associated with these two conditions [1].

Cancer is another condition that miRNAs were investigated in depth as possible biomarkers identifying different miRNAs which are differentially expressed in different cancer types, thus showing the specificity needed for a biomarker. It was suggested by Zhang et al. [2] that miRNAs can function as oncogenes as well as tumor suppressor genes since an upregulation of a specific miRNA can result to dysregulation of a tumor suppressor genes or my controlling genes of cell differentiation or apoptosis. Furthermore, a downregulation of specific miRNAs, such as let-7, which was detected in cancer, may lead to the overexpression of an oncogene. In addition, exosomal miRNAs in serum samples were detected to be differentially expressed in different cancer types, including breast cancer, pancreatic cancer as well as ovarian cancer [3]. In addition, miRNAs were also investigated for their association as therapeutic targets since by changing their expression the expression of their target genes ideally changes as well. Therefore, a change in miRNA and target gene expression is a promising approach for the modern medicine. Research is expanding on the development of synthetic miRNAs which could have similar functions with specific downregulated miRNAs in different disease [4]. Kreth et al. [4] has also summarized miRNA inhibitors that have been investigated in different diseases (T-cell Lymphoma, Hepatitis C). In particular, inhibitors may downregulate the expression of overexpressed miRNAs therefore increase the expression of their target genes. In a similar manner, inhibitors and synthetic miRNAs can be used to control the expression levels of miRNAs that act as oncogenes or tumor suppressor genes in cancer and consequently as therapeutic targets for the disease [5].

MicroRNAs introduce a new era in the identification as well as in the therapy of numerous diseases. Several studies have investigated and identified miRNAs which are differentially expressed in plasma/serum and/or tissue samples of different diseases indicating their role 
as possible biomarkers. Additionally, the target genes affected were also investigated in numerous cases identifying how could possibly be associated with different diseases. Therefore, at these results it is concluded that ideally, a panel with more than one miRNAs together with their validated target genes should be used for the identification of a specific disease since the expression of the miRNAs can also be affected by age, gender, diet as well as smoking and other factors. Regarding the role of miRNAs as therapeutic targets, it was investigated in depth. Studies have identified that by targeting miRNAs which target genes that play crucial roles in specific diseases may manage the severity of the disease.

\section{Conclusion}

In conclusion, by discussing briefly some of the data available on miRNAs it is shown that they can be both, biomarkers, as well as therapeutic targets. The identification of specific miRNAs which are dysregulated in different diseases, could lead to the discovery of new biomarkers and in addition these could be used as new therapeutic targets.

\section{References}

1. Zhou S, Jin JP, Wang JQ, Zhang ZG, Freedman JH, et al. (2018) miRNAS in cardiovascular diseases: potential biomarkers, therapeutic targets and challenges. Acta Pharmacologica Sinica 39: 1073-1084.

2. Zhang B, Pan X, Cobb GP, Anderson TA (2007) MicroRNAs as oncogenes and tumor suppressors. Dev Biol 302(1): 1-12.

3. Thind A, Wilson C (2016) Exosomal miRNAs as cancer biomarkers and therapeutic targets. J Extracell Vesicles 5(10): 31992.

4. Kreth S, Hübner M, Hinske LC (2018) MicroRNAs as clinical biomarkers and therapeutic tools in perioperative medicine. Anesth Analg 126(2): 670-681.

5. Rupaimoole R, Slack FJ (2017) MicroRNA therapeutics: towards a new era for the management of cancer and other diseases. Nat Rev Drug Discov 16(3): 203-222. 